\title{
Consensus over Ring Networks as a Quadratic Optimal Control Problem
}

\author{
J. A. Rogge ${ }^{*}$ J. A. K. Suykens ${ }^{* *}$ D. Aeyels ${ }^{*}$ \\ * Ghent University, SYSTeMS, Technologiepark Zwijnaarde 914, \\ B-9052 Gent, Belgium \\ (e-mail: \{jonathan.rogge,dirk.aeyels\}@ugent.be) \\ ** K. U. Leuven, ESAT-SCD, Kasteelpark Arenberg 10, B-3001 \\ Leuven, Belgium (e-mail: Johan.Suykens@esat.kuleuven.be)
}

\begin{abstract}
This paper presents the consensus problem in the framework of optimal control. Our aim is to synchronize a set of identical linear systems. We propose a cost which penalizes mutual differences between the states of these systems. The feedback matrix resulting from this linear quadratic control problem represents the interconnection network which synchronizes the systems. In general the interconnection structure is of the all-to-all type. We show that it is possible to devise an LQR problem in which the cost results in an interconnection structure representing ring coupling. Care has to be taken that the effect of the feedback control is restricted to synchronizing the systems, i.e. when the systems are synchronized, the feedback control signal is required to be equal to zero.
\end{abstract}

Keywords: synchronization, linear quadratic regulator, interconnected systems, algebraic Riccati equations, control system synthesis

\section{INTRODUCTION}

Over the last decade the research domain of cooperative control for multi-agent networked systems has experienced an on-going expansion of research activity. Multi-agent systems arise in areas where several autonomous systems are interacting with each other. Applications can be found in diverse areas such as interactions of local controllers in the power grid (Venkat et al. (2008)), formation control of autonomous vehicles (Fax and Murray (2004), OlfatiSaber and Murray (2004)), synchronization of oscillating systems (Rosenblum et al. (1996)), etc. Control of the multi-agent system arises through exchange of information between subsystems. The network of information exchange is represented by a graph whose vertices are the subsystems and whose edges constitute the allowed interaction channels between the subsystems.

An important subdomain of multi-agent systems research treats consensus problems. In this class of problems the subsystems share the goal of reaching a common objective. In many applications this corresponds to systems trying to reach the same state value. This value can be either timevarying or constant. When the subsystems exhibit oscillatory behavior, reaching consensus is called synchronization of the subsystems. Incorporating some adjustments (for instance, using different offsets), a consensus problem can produce a desired formation structure. An overview of consensus problems is given in Ren et al. (2005) and Ren and Beard (2008).

One line of recent research aims to extend synchronization results to systems with more general dynamics. In Scardovi and Sepulchre (2009), for instance, synchronization of identical linear systems under a dynamical coupling struc- ture is discussed. A second line of recent research studies consensus problems with limited interaction properties, (see e.g. Savkin (2006), Delvenne et al. (2009)). One wishes to limit interactions between systems in order to reduce a corresponding cost. In Delvenne et al. (2009) an optimum between interaction cost and control performance is obtained for a class of linear discrete-time systems.

A different viewpoint on control of networked systems is delivered by the linear quadratic (LQ) control framework. In this framework the interacting subsystems are necessarily linear. Traditionally this optimal control problem treats a single given linear system. After devising a cost which is quadratic in the state and the input of the system, the method delivers a linear stabilizing state feedback. In Levine and Athans (1970), Wenk and Knapp (1980), Gupta et al. (2005) the feedback matrix is assumed to be subject to structural constraints, such as for instance certain elements being zero. This assumption is relevant in a multi-agent networked system framework: given a linear multi-agent system, the feedback matrix produced by the linear quadratic control method represents the interaction topology between the subsystems. A zero-element in this matrix corresponds to the inability of one of the subsystem to influence a specific other one. When considering such constraints on the feedback matrix, the LQ method yields a sub-optimal solution compared to a feedback without restrictions. In Langbort and Gupta (2009) a method is described which adjusts the above problem formulation in order to obtain an optimal solution. The idea is to adjust the given matrix $Q$ representing the cost on the states in such a way that it yields an optimal feedback that is in correspondence with the given restrictions on it. This is an inverse problem, where the cost is synthesized starting 
from a desired feedback matrix. The proposed adjustments have a physical meaning: they represent extra costs on certain interconnection links.

The present paper combines the topic of restrictedinteraction consensus problems with the synthesis of a suitable cost function in the optimal control framework. The idea to use optimal control to obtain consensus or synchronization has been considered in Liu et al. (2009). However, in that paper it is a tracking control that drives the systems to consensus: the synchronized solution of each uncoupled subsystem is determined beforehand and the controller ensures that every system tracks this solution in an optimal way. In our approach synchronization is obtained through (optimal) interactions between the subsystems. We do not impose the synchronized solution a priori; we only require that the state differences between subsystems converge to zero. Up to our knowledge, this is a novel approach to the synchronization/consensus problem. Adopting the main idea of Langbort and Gupta (2009), which has also been proposed by Jovanovic (2010), our goal is to synthesize a quadratic cost for a linear multi-agent system consisting of identical systems, satisfying some given structural constraints. The idea of designing an LQR control satisfying structural constraints, is also used in Borrelli and Keviczky (2008), where the given constraints are related to stabilizability of the interconnected system. In the present paper the requirement of stabilizability is replaced by demanding the interconnected system reaches consensus. The constraints imposed are the following.

- The structure of the feedback matrix is chosen to correspond to a ring interconnection between the systems.

- The feedback matrix, resulting from the LQ problem realizes consensus between the subsystems.

- The feedback control is not allowed to affect the dynamics of the system when consensus is attained.

We decide to satisfy the first constraint by coupling the subsystems into a ring. The system under study then belongs to the class of spatially invariant systems. General results on optimal control of such systems are obtained in Bamieh et al. (2002) and Motee and Jadbabaie (2008). However, the assumptions on the cost in Bamieh et al. (2002) is more restrictive than the second and third constraint above allow. More precisely, we will show that the cost resulting in consensus is determined by a positive semi-definite matrix. This matrix is assumed to be positive definite in Bamieh et al. (2002). Furthermore, the results in Motee and Jadbabaie (2008) treat the spatial structure of optimal feedback controllers which are spatially decaying. In the present paper, however, feedback controllers coupling the systems in a ring are considered, which do not belong to the class of spatially-decaying controllers.

This paper is organized as follows. In Section 2 the problem of synthesizing an LQ cost that steers the system to consensus is defined for systems of general dimension. In Section 3 the desired cost is mathematically constructed in the case of scalar systems. Section 4 proves that this cost results in the desired feedback control. In Section 5 a simulation is presented where harmonic oscillators synchronize in an optimal fashion.

\section{PROBLEM STATEMENT}

In this section the synthesis problem is introduced. Consider $N$ uncoupled identical linear systems described by

$$
\dot{x}_{i}=\tilde{A} x_{i}+\tilde{B} u_{i}, \quad i \in\{1, \ldots, N\},
$$

with $\tilde{A} \in \mathbb{R}^{n \times n}, \tilde{B} \in \mathbb{R}^{n \times p}, x_{i}: \mathbb{R} \rightarrow \mathbb{R}^{n} ; t \mapsto x_{i}(t)$, and $u_{i}: \mathbb{R} \rightarrow \mathbb{R}^{p} ; t \mapsto u_{i}(t)$. This set of $N$ system equations can be written into one system equation:

$$
\dot{x}=A x+B u,
$$

where $A:=I_{N} \otimes \tilde{A} \in \mathbb{R}^{N n \times N n}, B:=I_{N} \otimes \tilde{B} \in \mathbb{R}^{N n \times N p}$, $x:=\left[x_{1}^{T} \cdots x_{N}^{T}\right]^{T}: \mathbb{R} \rightarrow \mathbb{R}^{N n}$, and $u:=\left[u_{1}^{T} \cdots u_{N}^{T}\right]^{T}:$ $\mathbb{R} \rightarrow \mathbb{R}^{N p}$. The symbol $\otimes$ denotes the Kronecker product or direct product, and $I_{N}$ is the $N \times N$ identity matrix.

As stated in the introductory section, we propose a feedback control

$$
u=K x
$$

representing ring coupling between the subsystems:

$u_{i}=K_{i, i-1} x_{i-1}+K_{i, i} x_{i}+K_{i, i+1} x_{i+1}, i \in\{1, \ldots, N\}$, (4) where $K_{i, j}$ are submatrices of $K$, with $K_{-1,1} \equiv K_{1, N}$, $K_{N, N+1} \equiv K_{N, 1}$ and $x_{-1} \equiv x_{N}, x_{N+1} \equiv x_{1}$.

This feedback control is demanded to be the solution of a linear quadratic control problem: the feedback (3) equals the input $\hat{u}: \mathbb{R} \rightarrow \mathbb{R}^{N p} ; t \mapsto \hat{u}(t)$ that satisfies

$$
\begin{aligned}
\hat{u}=\arg \min _{u} J\left(x_{0}, u\right) & \\
& :=\arg \min _{u} \int_{0}^{\infty} x^{T}(t) Q x(t)+u^{T}(t) R u(t) \mathrm{d} t,
\end{aligned}
$$

with $Q=Q^{T}$ positive semi-definite, $R=R^{T}$ positive definite, and where $x: t \mapsto x(t)$ is the solution of (2) corresponding to the input $u$ and the given initial state $x(0)=x_{0}$. It is a standard result (see for instance Kwakernaak and Sivan (1972)) that this input $\hat{u}$ is indeed a linear transformation of the state:

$$
\hat{u}=K x:=-R^{-1} B^{T} P x,
$$

with $P$ a solution of the algebraic Riccati equation

$$
A^{T} P+P A-P B R^{-1} B^{T} P=-Q .
$$

For reasons of simplicity, we set $R=I_{N p}$ in the remainder of this paper. Since $A$ and $Q$ are symmetric, it follows from (7) that $P$ is symmetric as well. From (6) it follows that $P$ adopts the structure of $K$ represented by (4). Combining these two conditions on the structure of $P$ shows that the matrix $P$ belongs to the class of block-circulant matrices, with symmetric submatrices. For a general treatment of block-circulant matrices the reader is referred to Davis (1979).

Next, the cost (5) must result in a feedback control which steers the system to a consensus solution defined by the consensus property

$$
x_{i}(t)=x_{j}(t), \quad \forall i, j \in\{1, \ldots, N\}, \quad \forall t \geq 0 .
$$

If the system state satisfies the consensus property, the cost depending on the states is required to be zero:

$$
x^{T}(t) Q x(t)=0 \Longleftrightarrow x_{i}(t)=x_{j}(t), \forall i, j \in\{1, \ldots, N\} .
$$

We call the LQ control problem with a Q-matrix satisfying (8), a synchronizing $L Q$ control problem (SLQR).

In a final constraint it is demanded that the effect of the feedback control is restricted to steering to consensus. The 
feedback control is not allowed to alter the subsystems' uncoupled behavior when consensus is attained. Consider (2) in closed loop:

$$
\dot{x}=(A+B K) x,
$$

We demand that when the system exhibits consensus behavior, the feedback signal is equal to zero:

$$
K x(t)=0 \Longleftrightarrow x_{i}(t)=x_{j}(t), \forall i, j \in\{1, \ldots, N\} .
$$

The main result of the present paper consists of a proof by construction of the following conjecture:

Conjecture 1. Consider $N$ linear systems (1) with $\tilde{A}, \tilde{B} \in$ $\mathbb{R}$. There exists a quadratic cost $J$ defined in (5) satisfying (8) that results in a feedback control $u=K x$ satisfying both (4) and (9), and steering the systems to consensus.

\section{SYNTHESIS OF THE OPTIMAL CONTROL PROBLEM FOR SCALAR SYSTEMS}

\subsection{Preliminary result}

The following lemma on circulant matrices, adopted from Davis (1979), introduces notation and is applied in the next section.

Lemma 2. Consider the block circulant matrix $C \in$ $\mathbb{R}^{N n \times N n}$ :

$$
C=\left[\begin{array}{cccc}
C_{1} & C_{2} & \cdots & C_{N} \\
C_{N} & C_{1} & \cdots & C_{N-1} \\
\vdots & \vdots & \vdots & \vdots \\
C_{2} & C_{3} & \cdots & C_{1}
\end{array}\right]=: \operatorname{circ}\left(C_{1}, C_{2}, \ldots, C_{N}\right),
$$

where $C_{i} \in \mathbb{R}^{n \times n}$, for all $i \in\{1, \ldots N\}$. The matrix $C$ can be block diagonalized into a matrix $\Lambda$ :

$$
\Lambda=\operatorname{diag}\left(\Lambda_{1}, \ldots, \Lambda_{N}\right),
$$

where the blocks $\Lambda_{i} \in \mathbb{C}^{n \times n}$ are given by

$$
\Lambda_{i}=C_{1}+\omega^{i-1} C_{2}+\omega^{2(i-1)} C_{3}+\ldots+\omega^{(N-1)(i-1)} C_{N},
$$

for all $i \in\{1, \ldots N\}$, with $\omega:=\exp (2 \pi j / N)$, where ' $j$ ' represents the imaginary unit.

\subsection{Consensus of scalar systems}

Consider (1) where the systems are scalar:

$$
\dot{x}_{i}=a x_{i}+b u_{i}, \quad i \in\{1, \ldots, N\},
$$

with $a \in \mathbb{R}, b \in \mathbb{R} \backslash\{0\}, x_{i}: t \mapsto x_{i}(t) \in \mathbb{R}, u_{i}: t \mapsto u_{i}(t) \in$ $\mathbb{R}$. The corresponding system $(2)$ has $A=a I_{N}, B=b I_{N}$. It follows immediately that $(A, B)$ is controllable, and hence stabilizable. With $R=I_{N}$, the general Riccati equation (7) changes into

$$
2 a P-b^{2} P^{2}=-Q .
$$

First, we postulate a feedback regulator $K \in \mathbb{R}^{N \times N}$ with the desired structure. Condition (9) implies that $K$ has a zero eigenvalue belonging to the eigenvector $\mathbf{1}_{N}:=\left[\begin{array}{lll}1 & \cdots & 1\end{array}\right]^{T}$. From $(6)$ it follows that $P$ possesses the same property, i.e. $P \mathbf{1}_{N}=0$. The remaining eigenvalues of $P$ need to be located in the right half complex plane to ensure that $P$ is a positive semi-definite solution of (11). Combining expression (4) with $K_{i, j} \in \mathbb{R}$, (6) with $B=b I_{N}, R=I_{N}$, and the condition of symmetry $P=P^{T}$, shows that $P$ has the form

with $\gamma \in \mathbb{R}$.
Theorem 3. The matrix $P \in \mathbb{R}^{N \times N}$ as defined by (12) is positive semi-definite if and only if $\gamma \geq 0$.

Proof. Applying Lemma 2 reveals that the eigenvalues $\lambda_{P i}$ of $P$ are given by

$$
\begin{aligned}
\lambda_{P i} & =\gamma-\frac{\gamma}{2}\left(\omega^{i-1}+\omega^{(N-1)(i-1)}\right), \\
& =\gamma-\frac{\gamma}{2}\left[\exp \left(\frac{2 \pi j(i-1)}{N}\right)+\exp \left(\frac{-2 \pi j(i-1)}{N}\right)\right], \\
& =\gamma\left[1-\cos \left(\frac{2 \pi(i-1)}{N}\right)\right],
\end{aligned}
$$

with $i \in\{1, \ldots, N\}$. The above calculation shows that all eigenvalues are larger or equal to zero, if and only if $\gamma \geq 0$.

We exclude the case $\gamma=0$ from the analysis for the obvious reason that this corresponds to uncoupled subsystems (10).

Second, we investigate if it is possible to determine a cost $J$, with $R=I_{N}$, which has $P$ as a solution. Diagonalizing $P$ yields $Q$ in diagonal form via (11): if $\tilde{P}:=T^{-1} P T$ is diagonal, then

$$
\begin{aligned}
2 a \tilde{P}-b^{2} \tilde{P}^{2} & =2 a T^{-1} P T-b^{2}\left(T^{-1} P T\right)^{2}, \\
& =T^{-1}\left(2 a P-b^{2} P^{2}\right) T, \\
& =-T^{-1} Q T,
\end{aligned}
$$

is diagonal as well. This implies that the eigenvalues $\lambda_{Q i}$ of $Q$ can be easily expressed in terms of the eigenvalues $\lambda_{P i}$ of $P$ :

$$
\lambda_{Q i}=b^{2} \lambda_{P i}^{2}-2 a \lambda_{P i}, \quad i \in\{1, \ldots, N\} .
$$

Using the expression for $\lambda_{P i}$ obtained in the proof of Theorem 3, the eigenvalues of $Q$ can be written as

$$
\begin{gathered}
\lambda_{Q i}=-2 a \gamma\left[1-\cos \left(\frac{2 \pi(i-1)}{N}\right)\right]+ \\
b^{2} \gamma^{2}\left[1-\cos \left(\frac{2 \pi(i-1)}{N}\right)\right]^{2}, \\
\lambda_{Q i}=\gamma\left[1-\cos \left(\frac{2 \pi(i-1)}{N}\right)\right] \times \\
{\left[-2 a+b^{2} \gamma\left(1-\cos \left(\frac{2 \pi(i-1)}{N}\right)\right)\right] .}
\end{gathered}
$$

Since $P \mathbf{1}_{N}=0$, it follows from (11) that $Q$ has zero row sum: $Q \mathbf{1}_{N}=0$. This implies one of the eigenvalues $\lambda_{Q i}$ is equal to zero. Indeed, expression (14) shows that $\lambda_{Q 1}=0$.

In order to define a realistic cost function, $Q$ needs to be positive semi-definite. This is established in the following theorem.

Theorem 4. Consider a set of $N$ scalar systems defined by (10), with $N \geq 3$. If each scalar system is stable when uncoupled, i.e. $a \leq 0$, then $Q$, determined by (11) and (12) with $\gamma>0$, is always positive semi-definite.

If the scalar systems are unstable, i.e. $a>0$, then $Q$ is positive semi-definite if and only if

$$
\gamma \geq \frac{2 a}{b^{2}}\left(1-\cos \left(\frac{2 \pi}{N}\right)\right)^{-1}
$$


Proof. Consider expression (14) denoting the eigenvalues of $Q$. As observed before, $\lambda_{Q 1}=0$. It remains to be investigated under which conditions the remaining eigenvalues are larger than or equal to zero.

The first factor of (14) is always positive for $i \neq 1$. If $a<0$ the second factor is positive, proving the first part of the theorem.

If $a>0, \lambda_{i} \geq 0, i \neq 1$ if and only if

$$
\begin{aligned}
\gamma & \geq \frac{2 a}{b^{2}}\left(1-\cos \left(\frac{2 \pi(i-1)}{N}\right)\right)^{-1}, \forall i \in\{1, \ldots, N\}, \\
& \Leftrightarrow \\
\gamma & \geq \frac{2 a}{b^{2}}\left(1-\cos \left(\frac{2 \pi}{N}\right)\right)^{-1},
\end{aligned}
$$

concluding the proof.

Summarizing, so far we have obtained a cost $J$, determined by matrices $Q$ and $R$, that has the matrix $P$ of the form (12) as a solution of the corresponding Riccati equation. Moreover, $Q$ is positive semi-definite with a zero eigenvalue belonging to the eigenvector $\mathbf{1}_{N}$.

However, we have not yet proved that $P$ is the solution of the Riccati equation which minimizes the cost $J$. There might be other positive semi-definite solutions with a smaller cost. This problem is tackled in the next section.

\section{OPTIMAL SOLUTIONS FOR THE SLQR PROBLEM}

Recall the standard result of LQ control as it is found in many textbooks (see e.g. Kwakernaak and Sivan (1972)).

Theorem 5. Consider an LQ control problem consisting of a linear system

$$
\dot{x}=A x+B u,
$$

and a cost $J$ as defined in (5), with $Q \geq 0$ and $R>0$.

If $(A, B)$ is stabilizable and $(Q, A)$ is observable then the corresponding Riccati equation (7) has exactly one positive semi-definite solution $P$, which is also positive definite. This solution yields a stabilizing feedback, via (3), which minimizes the cost $J$.

If $(A, B)$ is stabilizable and $(Q, A)$ is detectable then $(7)$ has exactly one positive semi-definite solution $P$, which yields a stabilizing feedback, minimizing $J$.

This theorem is thoroughly reviewed in Wonham (1968) and Lancaster and Rodman (1995). The latter reference presents an in-depth description of the Riccati equation and its properties.

Unfortunately, Theorem 5 is not always applicable to the SLQR problem of the present paper. First, we do not require a stabilizing feedback which drives all subsystems to the origin. Our goal is a synchronizing feedback, driving all systems to a consensus solution, which is not necessarily the origin. Second, the matrix $Q$ we propose is such that together with $A=a I_{N},(Q, A)$ is not necessarily detectable. Indeed, the nonobservable mode of the system corresponds to the space spanned by $\mathbf{1}_{N}$ and is given by the eigenvalue $a$. It follows that if and only if the scalar systems are asymptotically stable, i.e. $a<0,(Q, A)$ is detectable.
The remainder of this section is divided into the case where $(Q, A)$ is detectable, i.e. the subsystems (10) are asymptotically stable, and the case where $(Q, A)$ is not detectable, i.e. with unstable subsystems.

\subsection{Synchronization of asymptotically stable systems}

Before considering the case of asymptotically stable systems, it is useful to remark that even without coupling the systems reach consensus: they all converge to the origin independently. Therefore adding an interconnection structure to synchronize the systems seems somewhat pointless. However, in some situations it can be desirable to have the systems converge to a common state before they converge to the origin.

We consider (10) with $a<0$. As mentioned before, the nonobservable mode of the system is given by this value $a$. In Martensson (1971) the following lemma is presented.

Lemma 6 . Let $\lambda_{i}<0$ be a nonobservable mode of $(Q, A)$. Then there is no positive definite solution of (7).

Combining this result with Theorem 5 , it is concluded that when $a<0$ the SLQR problem of the present paper has exactly one solution $P \geq 0$, which is not positive definite.

It follows that the $P$-matrix of the form (12) with $\gamma>0$ minimizes the cost (5) with $R=I_{N}$ and $Q$ defined by (11).

\subsection{Synchronization of unstable systems}

The situation where $a>0$ is less straightforward. Consider the following lemma (see for instance Lancaster and Rodman (1995)).

Lemma 7. If $(A, B)$ is stabilizable then the Riccati equation (11) has at least one positive semi-definite solution $P \geq 0$.

The Riccati equation corresponding to the SLQR problem of the present paper possibly possesses multiple positive semi-definite solutions, among which our postulated matrix $P$ satisfying (12) resides. Is this solution $P$ also the one minimizing the cost $J$ ? The answer to this question can be found using the general analysis of the nondetectable case as constructed by Kučera (1972b). This analysis determines all positive semi-definite solutions of the Riccati equation, provided there exists a solution $P_{s}$ that yields an asymptotically stable close-loop system. We briefly review the method as presented in Kučera (1972b).

Introduce the matrix $M \in \mathbb{R}^{2 N \times 2 N}$ :

$$
M:=\left[\begin{array}{cc}
A & -B R^{-1} B^{T} \\
-Q & -A^{T}
\end{array}\right] .
$$

In the present paper it is assumed that $M$ is diagonalizable. All solutions of the corresponding Riccati equation are determined by the following lemma (Kučera (1972b)). Lemma 8. Let each eigenvector $v_{i} \in \mathbb{R}^{2 N}$ of $M$ be denoted by

$$
v_{i}=\left[\begin{array}{l}
x_{i} \\
y_{i}
\end{array}\right]
$$

with $x_{i}, y_{i} \in \mathbb{R}^{N}$

Every solution $P$ of (7) has the form

$$
P=Y X^{-1}
$$


where $N \times N$ matrices $X=\left[\begin{array}{ll}x_{1} & x_{2} \ldots x_{N}\end{array}\right]$ and $Y=$ $\left[\begin{array}{llll}y_{1} & y_{2} \ldots y_{N}\end{array}\right]$ (with $x_{i}$ and $y_{i}$ as introduced in (17)) are chosen such that $X^{-1}$ exists.

The next lemma presents a collection of properties of $M$ from Mårtensson (1971), Kučera (1972a,b), and Lancaster and Rodman (1995).

Lemma 9. With notation introduced in (17), the matrix $M$ defined by (16) has the following properties.

(1) If $\lambda_{i}$ is an eigenvalue of $M$, then so is $-\lambda_{i}$.

(2) The closed-loop system matrix $A-B R^{-1} B^{T} P$ yielded by a solution (18) has $N$ eigenvalues $\lambda_{i}$ of $M$ as its eigenvalues, with $x_{i}$ of (17) as their associated eigenvectors.

(3) If $(A, B)$ is stabilizable and $(Q, A)$ has no nonobservable modes with zero real part, then $M$ has no eigenvalues with zero-real part.

(4) The Riccati equation (7) has a solution $P \geq 0$ yielding an asymptotically stable closed-loop system if and only if $(A, B)$ is stabilizable and $\operatorname{Re} \lambda_{i} \neq 0$ for all eigenvalues $\lambda_{i}$ of $M$. This solution is denoted by $P_{s}$.

(5) Matrix $M$ has an eigenvector

$$
\left[\begin{array}{c}
x_{i} \\
0
\end{array}\right]
$$

associated with the eigenvalue $\lambda_{i}$ if and only if $\lambda_{i}$ is a nonobservable eigenvalue of $(Q, A)$.

Let us now introduce the sets $\mathcal{S}$ and $\mathcal{R}$. Let $\mathcal{S}$ denote the set of eigenvectors of $M$ of the form (19) which correspond to the nondetectable eigenvalues $\lambda_{i}$, i.e. $\operatorname{Re} \lambda_{i}>0$.

Let $\mathcal{R}$ denote the set of eigenvectors of $M$ of the form (17) which correspond to the eigenvalues of $M$ which are the opposite of the nondetectable eigenvalues.

From Lemma 9 it follows that the eigenvectors belonging to $\mathcal{R}$ are needed in the construction of $P_{s}$ according to (18).

Let $\mathcal{S}_{k} \subseteq \mathcal{S}$ and $\mathcal{R}_{k} \subseteq \mathcal{R}$ be two sets with the same number of elements. Consider the solution $P_{s}$ of (7) defined in Lemma 9(4). Starting from $P_{s}$, one can compute other solutions of (7). Write $P_{s}$ as $Y X^{-1}$ as described in (18) and consider a set $\mathcal{R}_{k}$. Replace the vectors $x_{i} \in X, y_{i} \in Y$ that correspond to the vectors $v_{i}$ in $\mathcal{R}_{k}$, with vectors corresponding to those in the set $\mathcal{S}_{k}$. Call the matrices obtained in this way $X_{k}$ and $Y_{k}$, and define $P_{k}:=Y_{k} X_{k}^{-1}$. It can be proved that $P_{k}$ is a solution of (7). Such a solution $P_{k}$ is said to be supported by the set $\mathcal{S}_{k}$.

The main theorem in Kučera (1972b) gives the following result on the positive semi-definite solutions of the Riccati equation.

Theorem 10. Suppose $M$ has no eigenvalues with zero-real part. Then the solutions $P_{k}$ supported respectively by all sets $\mathcal{S}_{k} \subseteq \mathcal{S}$ form the class of all positive semi-definite solutions of (11).

Combining this theorem with Lemma 9 (3) yields the following corollary.

Corollary 11. Suppose that $(A, B)$ is stabilizable and $(Q, A)$ has no nonobservable modes with zero real part. Then the solutions $P_{k}$ supported respectively by all sets $\mathcal{S}_{k} \subseteq \mathcal{S}$ form the class of all positive semi-definite solutions of $(\overline{7})$.
We are now in a position to determine all positive semidefinite solutions of (7) corresponding to the SLQR problem with $a>0$. The matrix $M$ corresponding to (10) is given by

$$
M=\left[\begin{array}{cc}
a I_{N} & -b^{2} I_{N} \\
-Q & -a I_{N}
\end{array}\right] .
$$

Since $Q$ is such that it has eigenvector $\mathbf{1}_{N}$ associated with eigenvalue $0, M$ has an eigenvector

$$
\left[\begin{array}{c}
\mathbf{1}_{N} \\
0
\end{array}\right]
$$

associated with the nondetectable mode $a>0$. The eigenvector of $M$ associated to $-a$ can be easily calculated as

$$
\left[\begin{array}{c}
\mathbf{1}_{N} \\
\frac{2 a}{b^{2}} \mathbf{1}_{N}
\end{array}\right]
$$

The sets $\mathcal{S}$ and $\mathcal{R}$ are singletons, which implies that there are two positive semi-definite solutions to the SLQR problem, one of which is the asymptotically stabilizing solution $P_{s}$. Call the remaining solution $P_{0}$.

Theorem 12. For the SLQR problem it holds that

$$
P_{s}=P_{0}+\frac{2 a}{b^{2} N} \mathbf{1}_{N} \mathbf{1}_{N}^{T} .
$$

Proof. It follows from Lemma 9 (2) and the above computed eigenvectors (20) and (21), that both closed-loop system matrices $A-B B^{T} P_{s}$ and $A-B B^{T} P_{0}$ have the same eigenvectors. Moreover, they have $N-1$ eigenvalues in common. The eigenvalue that is different, has the value $-a$ for $A-B B^{T} P_{s}$ and the value $a$ for $A-B B^{T} P_{0}$, and belongs to the eigenvector $\mathbf{1}_{N}$. Hence,

$$
\exists \alpha \in \mathbb{R}: P_{s}=P_{0}+\alpha \mathbf{1}_{N} \mathbf{1}_{N}^{T} .
$$

The value for $\alpha$ is obtained by imposing that both solutions $P_{s}$ and $P_{0}$ correspond to the same $Q$-matrix via the Riccati equation (11):

$$
-b^{2} P_{s}^{2}+2 a P_{s}=-b^{2} P_{0}^{2}+2 a P_{0} .
$$

Inserting (22) yields

$$
\begin{array}{r}
-b^{2}\left(P_{0}^{2}+\alpha \mathbf{1}_{N} \mathbf{1}_{N}^{T} P_{0}+\alpha P_{0} \mathbf{1}_{N} \mathbf{1}_{N}^{T}+\alpha^{2} \mathbf{1}_{N} \mathbf{1}_{N}^{T} \mathbf{1}_{N} \mathbf{1}_{N}^{T}\right) \\
+2 a P_{0}+2 a \alpha \mathbf{1}_{N} \mathbf{1}_{N}^{T}=-b^{2} P_{0}^{2}+2 a P_{0}
\end{array}
$$

Since $P_{0}=P_{0}^{T}$ and the row sum of $P_{0}$ is zero, this changes into

$$
\begin{aligned}
&-b^{2} \alpha^{2} N \mathbf{1}_{N} \mathbf{1}_{N}^{T}+2 a \alpha \mathbf{1}_{N} \mathbf{1}_{N}^{T} \Longleftrightarrow 0, \\
&-b^{2} \alpha^{2} N+2 a \alpha \Longleftrightarrow 0, \\
& \Longleftrightarrow \\
& \alpha=0 \text { or } \alpha=\frac{2 a}{b^{2} N},
\end{aligned}
$$

concluding the proof.

Theorem 13. The matrix $P_{0}$ corresponds to the feedback control minimizing the cost of the SLQR problem.

Proof. Let $J_{s}$ and $J_{0}$ be the values of the cost corresponding to $P_{s}$ and $P_{0}$ respectively. It is well-known that the size of the cost $J$ corresponding with a solution $P$ of the Riccati equation is given by

$$
J=\frac{1}{2} x_{0}^{T} P x_{0}
$$


where $x_{0}$ is the initial state of the system. Hence

$$
\begin{aligned}
J_{s} & =\frac{1}{2} x_{0}^{T} P_{s} x_{0}, \\
& =\frac{1}{2} x_{0}^{T} P_{0} x_{0}+\frac{a}{b^{2} N} x_{0}^{T} \mathbf{1}_{N} \mathbf{1}_{N}^{T} x_{0}, \\
& =\frac{1}{2} x_{0}^{T} P_{0} x_{0}+\frac{a}{b^{2} N}\left(\sum_{i=1}^{N} x_{i}\right)^{2}, \\
& >\frac{1}{2} x_{0}^{T} P_{0} x_{0}=J_{0} .
\end{aligned}
$$

\section{SIMULATION RESULTS: OPTIMAL SYNCHRONIZATION OF A SET OF HARMONIC OSCILLATORS}

In this section we consider a set of 10 harmonic oscillators defined by

$$
\ddot{x}_{i}+x_{i}=u_{i}, \quad i \in\{1, \ldots, 10\},
$$

which can be written equivalently as expression (1) with

$$
A_{i}=\left[\begin{array}{cc}
0 & 1 \\
-1 & 0
\end{array}\right], \quad B_{i}=\left[\begin{array}{l}
0 \\
1
\end{array}\right],
$$

where $i \in\{1, \ldots, 10\}$. For this system we synthesize a cost

$$
\int_{0}^{\infty} x^{T}(t) Q x(t)+u^{T}(t) u(t) \mathrm{d} t
$$

with positive semi-definite Q-matrix:

$$
\begin{array}{r}
Q=\operatorname{circ}\left(\left[\begin{array}{ll}
112 & 207 \\
207 & 417
\end{array}\right],\left[\begin{array}{cc}
-72 & -137.5 \\
-137.5 & -281
\end{array}\right],\left[\begin{array}{ll}
16 & 34 \\
34 & 72
\end{array}\right],\right. \\
\underbrace{0_{N}, \ldots, 0_{N}}_{5 \text { times }},\left[\begin{array}{ll}
16 & 34 \\
34 & 72
\end{array}\right],\left[\begin{array}{cc}
-72 & -137.5 \\
-137.5 & -281
\end{array}\right]),
\end{array}
$$

where $0_{N}$ denotes the $N \times N$ zero matrix.

The feedback control which minimizes this cost is given by

$$
u=K x=-B^{T} P x,
$$

with

$$
P=\operatorname{circ}(\left[\begin{array}{cc}
14 & 8 \\
8 & 17
\end{array}\right],\left[\begin{array}{cc}
-7 & -4 \\
-4 & -8.5
\end{array}\right], \underbrace{0_{N}, \ldots, 0_{N}}_{7 \text { times }},\left[\begin{array}{cc}
-7 & -4 \\
-4 & -8.5
\end{array}\right]) \text {, }
$$

and consequently

$$
K=\operatorname{circ}(\left[\begin{array}{ll}
-8 & -17
\end{array}\right],\left[\begin{array}{ll}
4 & 8.5
\end{array}\right], \underbrace{0_{N}, \ldots, 0_{N}}_{7 \text { times }},\left[\begin{array}{ll}
4 & 8.5
\end{array}\right]),
$$

expressing the ring coupling between the oscillators. The above values for $P$ and $Q$ are obtained by postulating a desired positive semi-definite $P$-matrix, calculating the corresponding $Q$-matrix according to (7), and checking if $Q$ satisfies all necessary conditions defined in Section 2 .

Figure 1 depicts the state trajectories of all 10 oscillators, starting from a random initial state. The horizontal axis indicates the position of each oscillator, the vertical axis indicates their velocity.

Figure 2 shows snapshots of the oscillators' states at different moments in time. The oscillators converge to a synchronizing behavior as follows. Starting from their initial conditions, the oscillator states first tend to align along a line with orientation top-left to bottom-right (see Figure 2 (b)). This alignment is not perfectly attained. While maintaining the alignment, the oscillator states circle around the origin with decreasing interdistances (Figure 2 (c)-(f)).

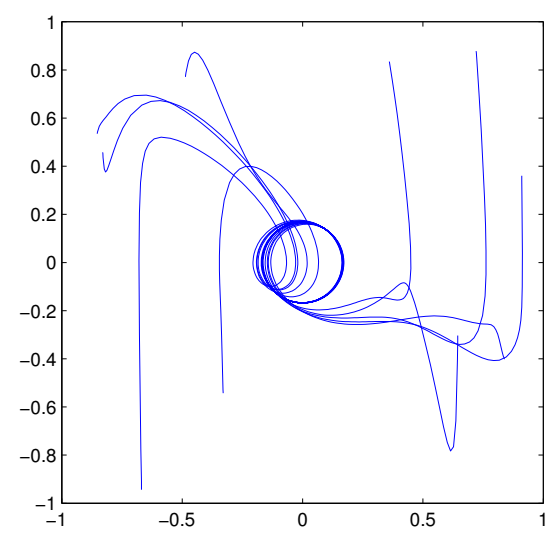

Fig. 1. The phase portrait of 10 synchronizing harmonic oscillators coupled in a ring.

\section{ACKNOWLEDGEMENTS}

The authors gratefully acknowledge the valuable comments of the anonymous referees.

This paper presents research results of the Belgian Network DYSCO (Dynamical Systems, Control, and Optimization), funded by the Interuniversity Attraction Poles Programme, initiated by the Belgian State, Science Policy Office. The scientific responsibility rests with its authors.

J. A. Rogge acknowledges support from IUAP DYSCO and CON4COORD, EU FP7 223844.

J. A. K. Suykens acknowledges support from K. U. Leuven, the Flemish government, FWO, and the Belgian federal science policy office (FWO G.0226.06, CoE EF/05/006, GOA MaNet, IUAP DYSCO, BIL/05/43)

\section{REFERENCES}

Bamieh, B., Paganini, F., and Dahleh, M.A. (2002). Distributed control of spatially invariant systems. IEEE Interactions on Automatic Control, 47(7), 1091-1107.

Borrelli, F. and Keviczky, T. (2008). Distributed LQR design for identical dynamically decoupled systems. IEEE Transactions on Automatic Control, 53(8), 1901-1912.

Davis, P.J. (1979). Circulant matrices. Chelsea Publishing.

Delvenne, J.C., Carli, R., and Zampieri, S. (2009). Optimal strategies in the average consensus problem. Systems $\&$ Control Letters, 58(10-11), 759-765.

Fax, J.A. and Murray, R.M. (2004). Information flow and cooperative control of vehicle formations. IEEE Transactions on Automatic Control, 49(9), 1465-1476.

Gupta, V., Hassibi, B., and Murray, R.M. (2005). A sub-optimal algorithm to synthesize control laws for a network of dynamic agents. International Journal of Control, 78(16), 1302-1313.

Jovanovic, M.R. (2010). On the optimality of localized distributed controllers. International Journal of Systems, Control and Communications, 2(1-3), 82-99.

Kučera, V. (1972a). A contribution to matrix quadratic equations. IEEE Transactions on Automatic Control, AC-17(3), 344-347.

Kučera, V. (1972b). On Nonnegative Definite Solutions to Matrix Quadratic Equations. Automatica, 8, 413-423. 


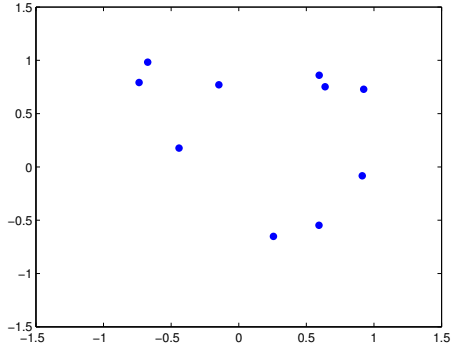

(a)

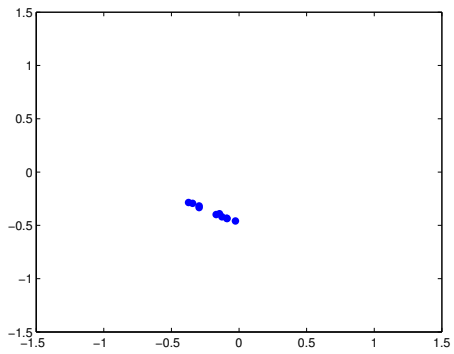

(d)

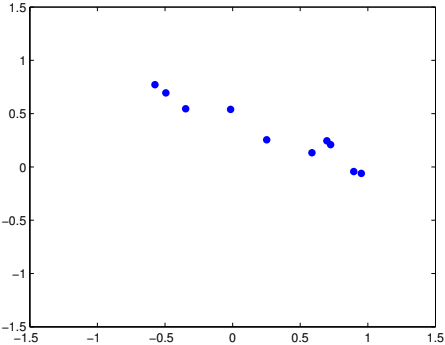

(b)

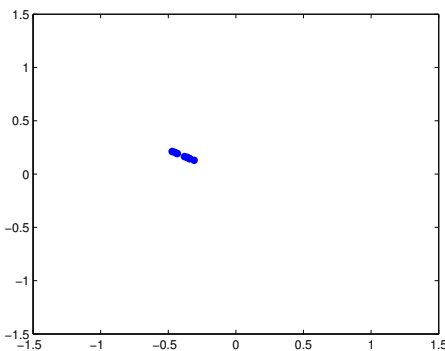

(e)

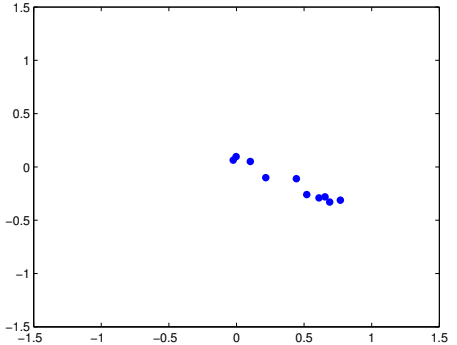

(c)

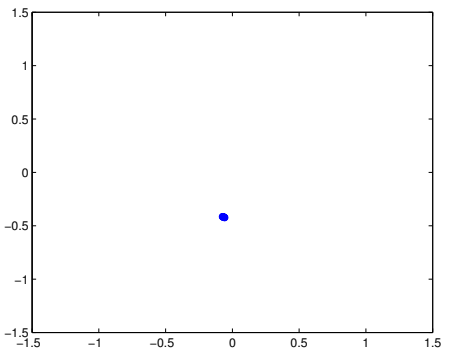

(f)

Fig. 2. Optimal synchronization of a group of 10 harmonic oscillators with ring coupling. Every dot represents the state of an oscillator. The frames are ordered (a) to (f) with increasing time instants.

Kwakernaak, H. and Sivan, R. (1972). Linear Optimal Control Systems. John Wiley and Sons, inc.

Lancaster, P. and Rodman, L. (1995). Algebraic Riccati Equations. Oxford University Press.

Langbort, C. and Gupta, V. (2009). Minimal interconnection topology in distributed control design. SIAM Journal on Control and Optimization, 48(1), 397-413.

Levine, W.S. and Athans, M. (1970). On determination of optimal constant output feedback gains for linear multivariable systems. IEEE Transactions on Automatic Control, AC-15(1), 44-48.

Liu, C., Duan, Z., Chen, G., and Huang, L. (2009). $\mathrm{L}_{2}$ norm performance index of synchronization and LQR control synthesis of complex networks. Automatica, 45(8), 1879-1885.

Motee, N. and Jadbabaie, A. (2008). Optimal control of spatially distributed systems. IEEE Transactions on Automatic Control, 53(7), 1616-1629.

Mårtensson, K. (1971). On the Matrix Riccati Equation. Information Sciences, 3, 17-49.

Olfati-Saber, R. and Murray, R.M. (2004). Consensus problems in networks of agents with switching topology and time-delays. IEEE Transactions on Automatic Control, 49(9), 1520-1533.

Ren, W. and Beard, R.W. (2008). Distributed Consensus in Multi-vehicle Cooperative Control: Theory and Applications. Springer.

Ren, W., Beard, R.W., and Atkins, E.M. (2005). A survey of consensus problems in multi-agent coordination. In Proceedings of the 2005 American Control Conference.

Rosenblum, M.G., Pikovsky, A.S., and Kurths, J. (1996). Phase synchronization of chaotic oscillators. Physical Review Letters, 76(11), 1804-1806.
Savkin, A.V. (2006). The problem of coordination and consensus achievement in groups of autonomous mobile robots with limited communication. Nonlinear Analysis - Theory, Methods \&S Applications, 65(5), 1094-1102.

Scardovi, L. and Sepulchre, R. (2009). Synchronization in networks of identical linear systems. Automatica, 45(11), $2557-2562$

Venkat, A.N., Hiskens, I.A., Rawlings, J.B., and Wright, S.J. (2008). Distributed MPC strategies with application to power system automatic generation control. IEEE Tansactions on Control Systems Technology, 16(6), 1192-1206.

Wenk, C.J. and Knapp, C.H. (1980). Parameter optimization in linear systems with arbitrarily constrained controller structure. IEEE Transactions on Automatic Control, AC-25(3), 496-500.

Wonham, W.M. (1968). On a matrix equation of stochastic control. SIAM Journal of Control, 6(5), 681-697. 